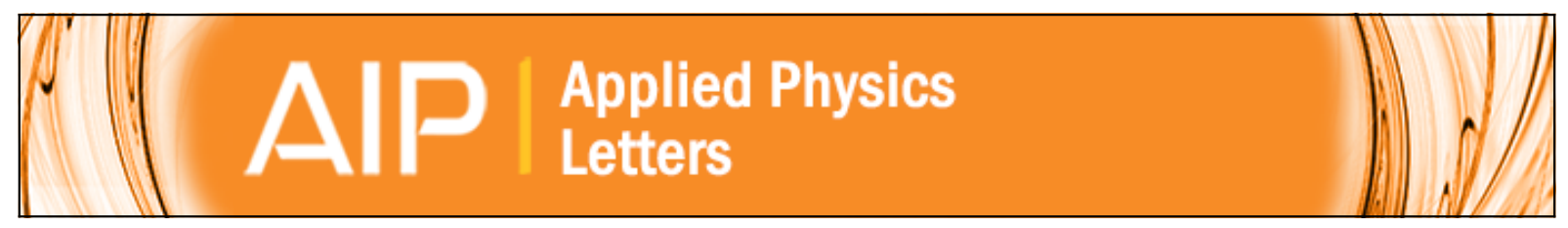

\title{
Polarization mode splitting in monolithic polymer microcavities
}

Luana Persano, Elisa Mele, Roberto Cingolani, and Dario Pisignano

Citation: Applied Physics Letters 87, 031103 (2005); doi: 10.1063/1.1994956

View online: http://dx.doi.org/10.1063/1.1994956

View Table of Contents: http://scitation.aip.org/content/aip/journal/apl/87/3?ver=pdfcov

Published by the AIP Publishing

\section{Articles you may be interested in}

Polarization resolved spatial near-field mapping of optical modes in an on-chip rolled-up bottle microcavity Appl. Phys. Lett. 105, 121106 (2014); 10.1063/1.4896544

Impact of disorder on high quality factor III-V nitride microcavities

Appl. Phys. Lett. 89, 261101 (2006); 10.1063/1.2420788

Widely tunable single-mode quantum cascade lasers with two monolithically coupled Fabry-Pérot cavities Appl. Phys. Lett. 89, 241126 (2006); 10.1063/1.2404933

Monolithic polymer microcavity lasers with on-top evaporated dielectric mirrors

Appl. Phys. Lett. 88, 121110 (2006); 10.1063/1.2179611

All-organic flexible polymer microcavity light-emitting diodes using $3 \mathrm{M}$ reflective multilayer polymer mirrors Appl. Phys. Lett. 87, 243504 (2005); 10.1063/1.2119416 


\title{
Polarization mode splitting in monolithic polymer microcavities
}

\author{
Luana Persano, ${ }^{\text {a) }}$ Elisa Mele, Roberto Cingolani, and Dario Pisignano \\ NNL, National Nanotechnology Laboratory of Istituto Nazionale di Fisica della Materia (INFM), and \\ Agilent Technologies Joint Lab c/o Dipartimento di Ingegneria dell'Innovazione, Università di Lecce, \\ via Arnesano, I-73100 Lecce, Italy
}

(Received 18 January 2005; accepted 13 June 2005; published online 11 July 2005)

\begin{abstract}
We demonstrate the mode splitting of the resonant emission from a symmetric monolithic organic semiconductor microcavity. The device, realized by low-temperature reactive electron-beam evaporation and deposition of a conjugated polymer, exhibits a $100 \mathrm{meV}$ polarization-induced splitting of the transmission and emission resonances for angles larger than $45^{\circ}$. This opens the way for the realization of novel polarized-emitting optoelectronic devices based on plastic materials.
\end{abstract}

(C) 2005 American Institute of Physics. [DOI: 10.1063/1.1994956]

The development of one-dimensional (1D) photonic crystals $(\mathrm{PhCs}),{ }^{1}$ and their integration in planar $^{2}$ and vertical ${ }^{3}$ microcavities provide an effective approach to control the light-matter interaction in solids, and to design innovative optical devices, such as fully plastic electroluminescent diodes. ${ }^{3}$ Vertical microcavities typically consist of two mirrors [either metallic single layers or distributed Bragg reflectors, ${ }^{3,4}$ (DBRs)] surrounding an optically active region of wavelength thickness. Such a structure induces the quantization of the electromagnetic field into a set of discrete confined photon modes. When the energy of the confined modes is resonant with an optical transition, it is possible to modify both the emission and the absorption spectra of the active material. For instance, the interaction between the excitonic and the confined photonic modes can give rise to the Rabi splitting of the resonant modes. ${ }^{5-7}$ Organic microcavities have been shown to operate in such a strong coupling regime, particularly by materials exhibiting large exciton oscillator strengths, and narrow linewidths, such as porphyrins, and $J$-aggregates of dye molecules. ${ }^{8,9}$ The strong coupling is identified by the energy anticrossing between the photonic and the excitonic modes. A doublet of states is observable at the minimum separation between the photon and the exciton modes, corresponding to cavity polariton modes separated by the Rabi splitting energy. The most of organic semiconductors generally show broadened spectra, because of the presence of vibronic progressions and the high degree of structural disorder, however Rabi splitting (up to $400 \mathrm{meV}$ at room temperature) has been reported on vertical microcavities based on conjugated polymers. ${ }^{10}$

Moreover, polarization-induced mode splitting has been recently observed in microcavities exploiting inorganic active media, such as InGaAs quantum dots ${ }^{11}$ and wells, ${ }^{12}$ and hydrogenated amorphous $\mathrm{Si}^{13}$ and very few works have shown a similar effect on polymers. ${ }^{14}$ Due to the relevant interest of microcavities for high-efficient light emitting diodes and vertical-cavity surface-emitting lasers, the development of novel architectures exhibiting polarization splitting has great importance for the realization of polarized optoelectronic devices.

In this letter, we report a study of a monolithic symmetric organic semiconductor microcavity structure, whose mirrors are realized entirely by reactive electron-beam evapora-

${ }^{a)}$ Electronic mail: luana.persano@unile.it tion of a few couples of $\mathrm{TiO}_{x}$ and $\mathrm{SiO}_{x}$ thin films at low temperature ( $T$ between 20 and $70{ }^{\circ} \mathrm{C}$ ). This technique definitely enables the fabrication of molecular optoelectronics in a unique and continuous high-vacuum deposition process. This procedure allows one to overcome problems of irreversible loss of adhesion between the organic compound and the substrate during deposition. In addition, it avoids the strong deterioration of the optical properties of the active organic layers, ${ }^{15}$ which is induced by the high temperature and the oxygen atmosphere usually employed to deposit high reflective oxide-based dielectric mirrors. A doublet is observed in the photoluminescence (PL) of our microcavities at detection angles larger than $45^{\circ}$. We found that the high-energy peak is transversal-magnetic (TM) polarized and the low energy one is transversal-electric (TE) polarized, thus assigning the observed doublet to polarization splitting.

The scheme of our microcavities is shown in Fig. 1(a). The bottom DBR, deposited onto Corning glass substrates by low-temperature electron-beam evaporation, is composed by $9.5 \lambda / 4$ pairs of $\mathrm{SiO}_{x}\left(d_{1}=95 \mathrm{~nm}\right)$ and $\mathrm{TiO}_{x}\left(d_{2}=70 \mathrm{~nm}\right)$ layers, that were used as low- $\left(n_{1}=1.42\right)$ and high- $\left(n_{2}\right.$ $=1.98)$ refractive index medium, respectively. The oxide films were deposited from $99.9 \%$ purity $\mathrm{TiO}_{2}$ tablets and $\mathrm{SiO}_{2}$ disks (Leybold, Germany) at a working pressure of $2.4 \times 10^{-4}$ mbar. A controlled oxygen atmosphere was accomplished by a gas inlet through a precision needle valve at

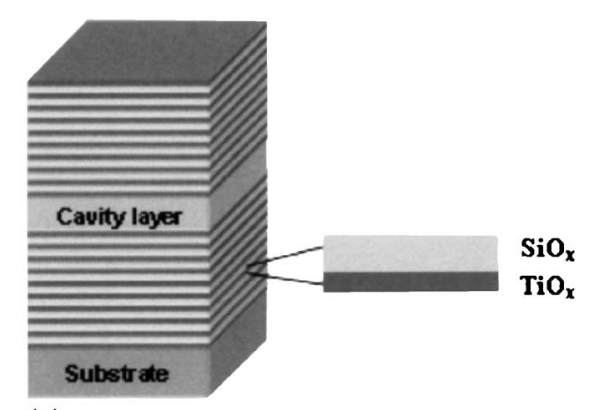

(a)

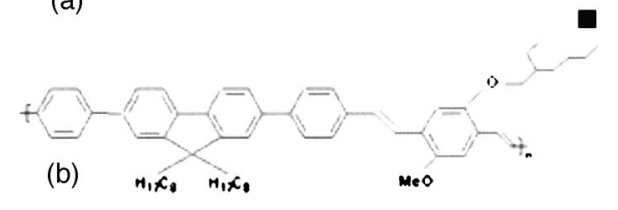

FIG. 1. Scheme of the microcavity structure (a) and molecular structure of the employed conjugated polymer (b). 


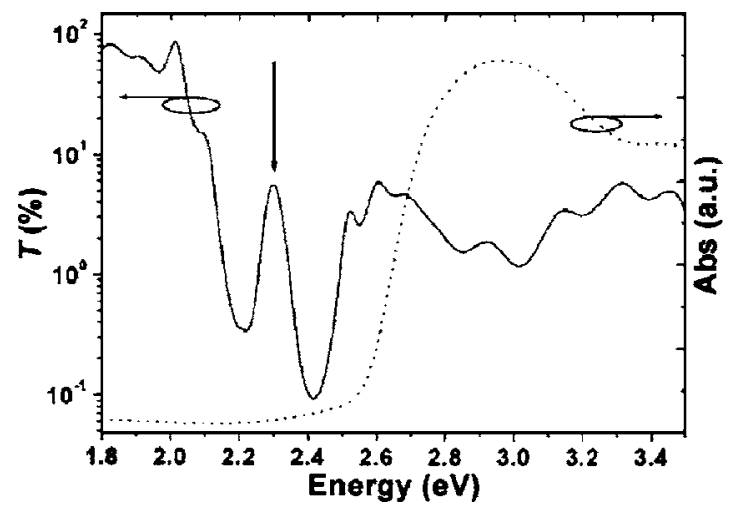

FIG. 2. Transmission spectrum of the complete microcavity (continuous line, vertical scale on the left) and absorption spectrum of a neat copolymer thin film (dotted line, vertical scale on the right). The arrow indicates the resonant mode within the microcavity stop band.

few $\mathrm{cm}$ from the samples. The deposition process was carried out at low $T$, the only heat source being the electrongun. An active organic semiconductor layer of the conjugatedcopolymer, poly[(9,9-dioctylfluorenyl-2,7-diyl)-co-(1,4diphenylene-vinylene-2-methoxy-5-\{2-ethylhexyloxy\}-benzene) $][(\mathrm{PFV})$ molecular structure in Fig. 1(b), American Dye Source, Canada] with a thickness, $L_{c}=360 \mathrm{~nm}$, was directly spin cast from chloroform solution onto the bottom DBR. The PFV PL spectrum is extended between 1.77 and $2.63 \mathrm{eV}$. The top mirror, composed by 9.5 periods of $\mathrm{TiO}_{x} /$ $\mathrm{SiO}_{x}$ layers, was then evaporated on the cavity layer at low $T$. The PL from the microcavity was measured by exciting the samples with a Xe light source (SP Spectral Products, $\mathrm{CT}$ ), centering the pump wavelength at $480 \mathrm{~nm}$ by a monochromator, and collecting the emission in a small solid angle $\left(\cong 10^{-3} \mathrm{rad}\right.$ ) around each viewing angle, $\Theta$, by a fibercoupled charge coupled device (Ocean Optics, FL). Angleresolved reflectance and normal incidence transmittance measurements were performed by a double-beam Cary 5000 spectrophotometer (Varian, Australia).

The normal incidence transmittance spectrum of the complete microcavity, together with the absorbance spectrum of a PFV film, are reported in Fig. 2 (continuous and dotted lines, respectively). The resonant mode of the microcavity is visible as a peak within the DBR stop band (arrow at 2.3 $\mathrm{eV}$ ), exhibiting a cavity quality factor, $Q \approx 40$. At higher energies $(>2.5 \mathrm{eV})$, the transmittance spectrum of the microcavity is of course modulated by the absorption of the conjugated polymer.

Figure 3 shows the reflectance $(R)$ and the PL spectra of the device, at different detection angles $\left(35^{\circ} \leqslant \Theta \leqslant 65^{\circ}\right)$. Besides the resonance at high energy, the lower-order emission peak can be clearly observed (though with a lower quality factor), at $2.17 \mathrm{eV}$ in the PL spectrum collected at $35^{\circ}$. Upon increasing the viewing angle, the high-energy resonance shifts well beyond the high-energy tail of the luminescence from PFV, so being no longer observable in the PL spectra. Differently, the angular dynamics of the lower-order resonance can be followed both in the $R$ and in the emission spectra also for angle up to $75^{\circ}$ : The energy, $E$, of the such a cavity photon mode remarkably increases upon increasing the detection angle $\Theta$, according to the dispersion relation: ${ }^{6}$

$$
E(\Theta)=E\left(1-\sin ^{2} \Theta / n_{\mathrm{eff}}^{2}\right)^{-1 / 2},
$$

where $n_{\text {eff }}$ indicates the effective refractive index of the structure. At $\Theta>45^{\circ}$, this mode splits, as seen in the double-

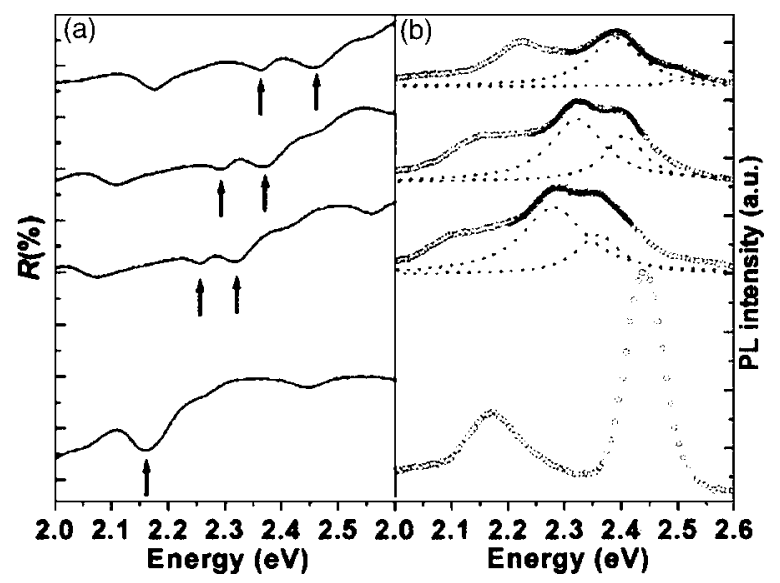

FIG. 3. Reflectance (a, continuous lines) and PL (b, empty-dots curves) spectra of the microcavity at different detection angles. (From bottom to top: $35^{\circ}, 50^{\circ}, 55^{\circ}$, and $65^{\circ}$.) The arrows in (a) indicate the mode splitting upon increasing viewing angle. In (b), we superimposed the best fit to experimental data in the splitting region (single curves: Dotted lines, superposition: Continuous line).

maximum PL and in the two dips in the reflectance spectra. In particular, the two-fold band of the PL spectra can be well fitted by a superposition of two separate lines [Fig. 3(b)]. From this fit, we extracted the angular dependence of the individual resonance modes (Fig. 4). The resonance splitting occurs at $\Theta=48^{\circ}$, growing nonlinearly from 80 to $100 \mathrm{meV}$ upon increasing the detection angle.

In particular, we found that the dependence of the central resonance energy of the splitting branches on the viewing angle is not consistent with the occurrence of Rabi splitting. ${ }^{8,16,17}$ In fact, the main signature of strongly coupled excitonic-photonic emission is not observed in our spectra. For Rabi splitting, the separation of the anticrossing polariton branches should be observed at any detection angle, giving rise to an energy of minimum splitting (i.e., the Rabi energy), and the photonlike mode should approach the excitonic absorption energy upon increasing the emission angle. Indeed, such strong-coupling regime is strongly favored only in microcavities which are based on active compounds with a single narrow optical transition.

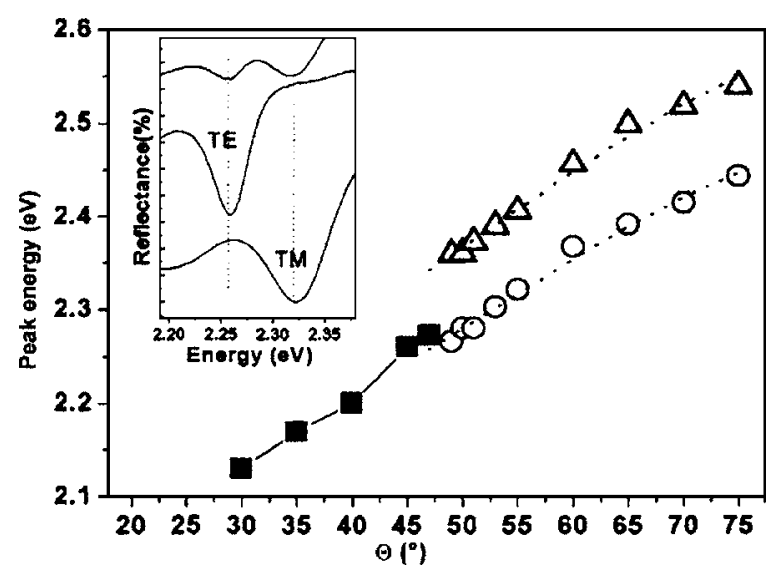

FIG. 4. Angular dependences of the resonance frequencies, extracted by PL measurements. Full squares: Main peak $\left(30^{\circ} \leqslant \Theta \leqslant 47^{\circ}\right)$, empty circles: TE peak, empty triangles: TM peak $\left(49^{\circ} \leqslant \Theta \leqslant 75^{\circ}\right)$. Dotted lines represent the fitting curves calculated according to Eq. (1). The inset shows the reflectance spectra from top to bottom: with unpolarized light, TE, and TM polarization. (Detection angle: $50^{\circ}$.) 
On the contrary, the here observed tuning of the cavity modes is due to a phenomenon entirely related to photon states. In order to gain a deeper understanding, we performed polarized angle-resolved measurements, finding that the high-energy splitting peak is TM polarized and the lowenergy one is TE polarized (inset of Fig. 4). Therefore, we attribute the doublet to TM-TE splitting, depending on the different behavior of the microcavity under different polarization conditions. In particular, the penetration depth of light inside the deposited DBR, $L_{\mathrm{DBR}}$, is strongly affected by the light polarization, increasing upon increasing angle for TM modes, and decreasing upon increasing angle for TE polarization. ${ }^{12}$ If we consider for simplicity the case of an empty microcavity, the cavity-mode frequency, $\omega_{m}$, can be expressed as:

$$
\omega_{m}(\Theta)=\frac{L_{c} \omega_{c}(\Theta)+L_{\mathrm{DBR}}(\Theta) \omega_{s}(\Theta)}{L_{\mathrm{eff}}(\Theta)},
$$

where $\omega_{c}=2 \pi c / n_{c} L_{c} \cos \Theta_{c}$ [with $\left.\Theta_{c}=\arcsin \left(\sin \Theta / n_{c}\right)\right]$ is the Fabry-Perot frequency under the approximation of negligible phase delay in the mirrors, and $\omega_{s}$ is the central frequency of the stop band of the DBR. For low refractiveindex contrasts, the polarization-induced splitting is controlled by the mismatch between $\omega_{s}$ and $\omega_{c}$, since it can be expressed as:

$$
\begin{aligned}
\omega_{m}^{\mathrm{TM}}(\Theta)-\omega_{m}^{\mathrm{TE}}(\Theta) \cong & A\left[L_{\mathrm{DBR}}^{\mathrm{TM}}(\Theta)-L_{\mathrm{DBR}}^{\mathrm{TE}}(\Theta)\right] \\
& \times\left[\omega_{s}(0)-\omega_{c}(0)\right],
\end{aligned}
$$

where $A>0$. Consequently, the TM mode can have lower or higher energy with respect to the TE one, depending on the mutual values of $\omega_{c}(0)$ or $\omega_{s}(0)$ : If $\omega_{s}>\omega_{c}$, one has $\omega_{m}^{\mathrm{TM}}(\Theta)>\omega_{m}^{\mathrm{TE}}(\Theta)$ (this occurs when the DBR period is larger than half-wavelength, $d_{1}+d_{2}>\lambda / 2$ ), whereas, if $\omega_{s}$ $<\omega_{c}\left(d_{1}+d_{2}<\lambda / 2\right)$, one has $\omega_{m}^{\mathrm{TM}}(\Theta)<\omega_{m}^{\mathrm{TE}}(\Theta)$. In our experiment, $\quad \omega_{s}(0)=3.62 \pi c \times 10^{-3} \mathrm{~nm}^{-1}>\omega_{c}(0)=3.26 \pi c$ $\times 10^{-3} \mathrm{~nm}^{-1}$, hence the TM mode has higher energy, as expected since the overall period of the employed DBRs (165 $\mathrm{nm}$ ) is smaller than a half-wavelength (about $300 \mathrm{~nm}$ ). This is clearly shown in the inset of Fig. 4, displaying the reflectance doublet of the microcavity and the TE (at $2.25 \mathrm{eV}$ ) and TM (at $2.32 \mathrm{eV}$ ) modes selected by polarized incident light. Finally, from the angular dependence of the two resonances, we extracted the effective refractive indexes of the modes. According to Eq. (1), $n_{\text {eff }}=1.79$ and 1.76 for the TE and the TM modes, respectively (fitting lines in Fig. 4).
In conclusion, low-temperature electron-beam deposition was used to fabricate novel monolithic microcavities based on a conjugated polymer. We found that a doublet is observed in both the reflectance and the PL spectra of our microcavities at angles larger than $45^{\circ}$. We attribute this effect to TM-TE polarization splitting, related to the different behavior of the microcavities under polarization conditions. The realization of light-interactive organic microcavities opens the way to the realization of a wide class of more complex optoelectonic devices, based on the interaction of photonic-band gap structured materials and organic semiconductors.

${ }^{1}$ J. D. Joannopoulos, R. D. Meade, and J. N. Winn, Photonic Crystals: Molding the Flow of Light (Princeton University Press, Cambridge, MA 1995); E. Yablonovitch, Phys. Rev. Lett. 58, 2059 (1987).

${ }^{2}$ G. A. Turnbull, P. Andrews, M. J. Jory, W. L. Barnes, and I. D. W. Samuel, Phys. Rev. B 64, 125122 (2001); D. Pisignano, L. Persano, E. Mele, P. Visconti, R. Cingolani, G. Gigli, G. Barbarella, and L. Favaretto Opt. Lett. 30, 260 (2005).

${ }^{3}$ P. K. H. Ho, S. Thomas, R. H. Friend, and N. Tessler, Science 285, 233 (1999); R. B. Fletcher, D. G. Lidzey, D. D. C. Bradley, M. Bernius, and S. Walker, Appl. Phys. Lett. 77, 1262 (2000); M. S. Unlu and S. Strite, J. Appl. Phys. 78, 607 (1995).

${ }^{4}$ V. Bulović, V. G. Kozlov, V. B. Khalfin, and S. R. Forrest, Science 279, 553 (1998).

${ }^{5}$ C. Weisbuch, M. Nishioka, A. Ishikawa, and Y. Arakawa, Phys. Rev. Lett. 69, 3314 (1992).

${ }^{6}$ M. S. Skolnick, T. A. Fisher, and D. M. Whittaker, Semicond. Sci. Technol. 13, 645 (1998).

${ }^{7}$ T. B. Norris, J.-K. Rhee, C.-Y. Sung, Y. Arakawa, M. Nishioka, and C. Weisbuch, Phys. Rev. B 50, 14663 (1994).

${ }^{8}$ D. G. Lidzey, D. D. C. Bradley, M. S. Skolnick, T. Virgili, S. Walker, and D. M. Whittaker, Nature (London) 395, 53 (1998).

${ }^{9}$ D. G. Lidzey, D. D. C. Bradley, T. Virgili, A. Armitage, M. S. Skolnick, and S. Walker, Phys. Rev. Lett. 82, 3316 (1999).

${ }^{10}$ N. Takada, T. Kamata, and D. D. C. Bradley, Appl. Phys. Lett. 82, 1812 (2003).

${ }^{11}$ C. Y. Hu, H. Z. Zheng, J. D. Zhang, H. Zhang, F. H. Yang, and Y. P. Zeng, Appl. Phys. Lett. 82, 665 (2003).

${ }^{12}$ G. Panzarini, L. C. Andreani, A. Armitage, D. Baxter, M. S. Skolnick, V. N. Astratov, J. S. Roberts, A. V. Kavokin, M. R. Vladimrova, and M. A. Kaliteevsky, Phys. Rev. B 59, 5082 (1999).

${ }^{13}$ A. A. Dukin, N. A. Feoktistov, V. G. Golubev, A. V. Medvedev, A. B. Pevtsov, and A. V. Sel'kin, Phys. Rev. E 67, 046602 (2003).

${ }^{14}$ T. Virgili, D. G. Lidzey, D. D. C. Bradley, and S. Walker, Synth. Met. 116, 497 (2001); Q. Song, L. Liu, T. Ling, L. Xu, and W. Wang, Appl. Phys. Lett. 82, 2939 (2003).

${ }^{15}$ M. Yan, L. J. Rothberg, F. Papadimitrakopoulos, M. E. Galvin, and T. M. Miller, Phys. Rev. Lett. 73, 744 (1994), and references therein.

${ }^{16}$ D. G. Lidzey, A. M. Fox, D. Rahn, M. S. Skolnick, V. M. Agranovich, and S. Walker, Phys. Rev. B 65, 195312 (2002).

${ }^{17}$ L. G. Connolly, D. G. Lidzey, R. Buttè, A. M. Adawi, D. M. Whittaker, M. S. Skolnick, and R. Airey, Appl. Phys. Lett. 83, 5377 (2003). 